\title{
Right ventricular cardiovascular magnetic resonance imaging: normal anatomy and spectrum of pathological findings
}

\author{
Nicola Galea • Iacopo Carbone • David Cannata • \\ Giuseppe Cannavale • Bettina Conti • Roberto Galea • \\ Andrea Frustaci • Carlo Catalano • Marco Francone
}

Received: 11 July 2012 /Revised: 27 November 2012 / Accepted: 10 January 2013 /Published online: 8 February 2013

(C) The Author(s) 2013. This article is published with open access at Springerlink.com

\begin{abstract}
Background The right ventricle (RV) has been defined as the "forgotten chamber", as its role in cardiac physiopathology has long been underestimated. Nevertheless, the RV is involved in a wide range of pathological conditions and its altered function may significantly affect the patient's clinical status.

Methods A selection of the most common cardiovascular magnetic resonance (CMR) features in a spectrum of pathological conditions is illustrated. Although its complex morphology, thin myocardium and trabeculated apex, RV can be accurately imaged by CMR, revealing its involvement in ischaemic and nonischaemic heart disease. CMR has emerged as the pre-eminent modality in monitoring ventricular performance in congenital heart disease, pulmonary hypertension and cardiomyopathies. Arrhythmogenic right ventricular cardiomyopathy is a difficult diagnosis and the recently revised task force criteria confirmed a crucial role of CMR to increase diagnostic accuracy, by combining detection of RV dilation, regional wall motion and structural abnormalities. Moreover, a multiparametric approach
\end{abstract}

Electronic supplementary material The online version of this article (doi:10.1007/s13244-013-0222-3) contains supplementary material, which is available to authorized users.

N. Galea $\cdot$ I. Carbone $\cdot$ D. Cannata $\cdot$ G. Cannavale $\cdot$ B. Conti $\cdot$

C. Catalano $\cdot$ M. Francone

Policlinico Umberto I, Department of Radiological, Oncological and

Pathological Sciences, Sapienza University of Rome, Rome, Italy

R. Galea $\cdot$ A. Frustaci

Policlinico Umberto I, Department of Cardiovascular Respiratory

Sciences, Sapienza University of Rome, Rome, Italy

N. Galea $(\triangle)$

Department of Radiological, Oncological and Pathological

Sciences, Sapienza University of Rome, Viale Regina Elena 324,

00161 Rome, Italy

e-mail: nicola.galea@gmail.com of CMR is often necessary for delineation and characterisation of cardiac masses.

Conclusion CMR, combining assessment of morphology, structure and function, has definitively emerged as the reference technique to evaluate a large variety of RV diseases. Teaching Points

- CMR offers unique advantages for imaging of many $R V$ congenital, ischaemic and non-ischaemic diseases.

- Because of high reproducibility, CMR has a crucial role in decision-making for chronic RV pathology.

- The use of CMR increases detection of $R V$ disease as infarction or arrhythmogenic cardiomyopathy.

Keywords Cardiovascular magnetic resonance $\cdot$ Right ventricle $\cdot$ Ischaemic heart disease $\cdot$ Non-ischaemic cardiomyopathy $\cdot$ Congenital heart disease

\section{Introduction}

The right ventricle (RV) is involved in a wide variety of acquired and congenital heart diseases, even though the primary diagnostic focus is attributed to the left ventricle (LV) in most clinical conditions. [1] The importance of RV pathology and dysfunction has been highlighted in several recent publications in terms of impact on a patient's prognosis and therapeutic management [1-4].

Cardiovascular magnetic resonance (CMR) has emerged as the referenced standard technique for this purpose due to its unique capability in providing an accurate and reproducible assessment of function and tissue characterisation $[5,6]$.

Although trans-thoracic echocardiography is recommended as an initial imaging instrument, it has limited diagnostic capabilities for the assessment of RV due to its thin wall, peculiar morphology and the anterior position in the chest $[7,8]$. 
The present pictorial review of the CMR features will illustrate RV anatomy and its range of various pathological conditions, thus leading to a more prompt recognition of disease.

\section{CMR protocol}

All CMR exams were performed with a 1.5-T unit (Avanto; Siemens, Erlangen, Germany) with an eight-element phasedarray coil.

The standard CMR protocol included T2-weighted blackblood short-inversion time turbo spin-echo imaging (T2WI), cine balanced steady-state free-precession sequences (cine SSFP) and T1-weighted contrast-enhanced inversion recovery segmented gradient echo sequence (LGE-T1WI) acquired 10$15 \mathrm{~min}$ after the intravenous administration of $0.2 \mathrm{mmol} / \mathrm{kg}$ gadobenate dimeglumine (Gd-BOPTA) for the evaluation of the late gadolinium enhancement (LGE) (see the online-only "Electronic supplementary material" for details).

Morphological T1-weighted TSE black blood sequences were added to the above protocol only for specific indications (e.g. morphology assessment and tissue characterisation in suspected arrhythmogenic right ventricular cardiomyopathy [ARVC]).

Flow measurement using velocity-encoded phase-contrast technique is a pre-eminent strength of CMR, enabling the detection and quantification of any valvular, vessel or conduit stenosis or regurgitation, whose main application is in the assessment of congenital heart disease.

Due to the complex anatomy of the RV, a combination of imaging in different cardiac planes is always helpful to better depict cardiac structures and therefore all sequences were performed on short axis, four-chamber and RV inflowoutflow views.

\section{Normal anatomy of right ventricle}

Recognising typical features of each cardiac chamber, in particular the ventricles, is necessary to interpret cardiac morphology, which may not be straightforward in some pathological conditions such as complex congenital heart diseases.

$\mathrm{RV}$ morphology is characterised as pyramidal in shape with an inlet portion communicating to the right atrium through the tricuspid valve, an outlet or infundibular portion continuous to the pulmonary valve and a trabecular apex (Fig. 1).

Some features helpful in recognising the morphological $\mathrm{RV}$ are:

- Chordal attachment of septal leaflet of tricuspid valve to the ventricular septum (never present in LV anatomy)

- Presence of the moderator band, a muscular structure connecting the apical ventricular septum with the apical
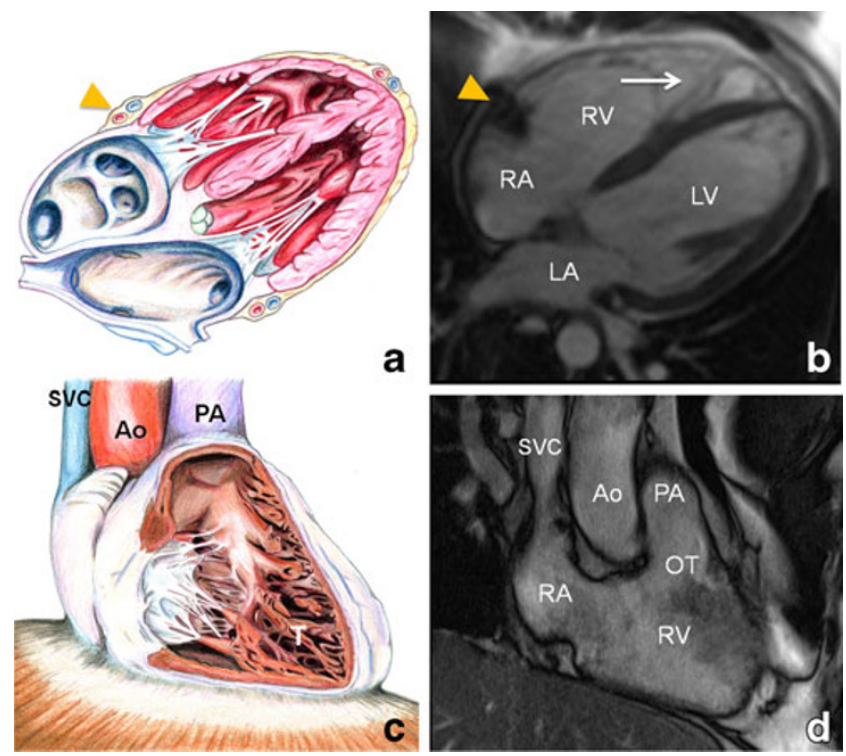

Fig. 1 Freehand drawings $(\mathbf{a}, \mathbf{c})$ and corresponding cine SSFP images on four-chambers (b) and three-chambers (d) views demonstrating normal RV anatomy: moderator band (arrow), tendinous cords of tricuspid valve and trabeculations $(T) . R A$ right atrium, $R V$ right ventricle, $L A$ left atrium, $L V$ left ventricle, orange triangle right atrioventricular groove containing the right coronary artery and small cardiac vein, $S C V$ superior vena cava, $A o$ aorta, $P A$ pulmonary artery, $O T$ right ventricular outflow tract

part of the RV free wall, involved in the cardiac electrical conduction system

- "Crista supraventricularis", a ridge separating the infundibulum and the inlet part

- A trabeculated RV apex

Notably, there is no muscular or chordal structural inserts on the septum in LV; thus the moderator band and tricuspidal chordal attachment may be very helpful in distinguishing the morphological RV from the LV in congenital malformation.

Conversely, the interventricular septum shape and lateral wall thickness are not reliable features in defining the morphology of the RV.

In a normal subject, the chamber equals the LV in volume while the ventricular wall and trabeculations are substantially thinner than those of the LV (RV wall thickness: 3$5 \mathrm{~mm}$ ), reflecting the different pressure regimen.

\section{Congenital heart disease}

The RV is often abnormal in congenital heart disease, in both isolated and complex malformations.

In this clinical setting, CMR has rapidly become the standard reference, whereas echocardiography suffers a lack of optimal acoustic window [9], particularly in postoperative patients, and CMR is independent of geometrical assumptions. 
CMR has become a valid and comprehensive tool to define the anatomy, to assess bi-ventricular function [6] and haemodynamics, and to measure blood-flow by using phase contrast technique. Information provided by the exam can play a crucial role either in establishing the need and type of treatment or during follow-up after correction. For example, assessing the haemodynamic outcome after surgery requires a reproducible and accurate assessment of ventricular function as clinical decisions are usually based on a change in serial measurements rather than a single absolute value [10].

The most common congenital conditions at risk for progressive right ventricular dilatation and dysfunction are pulmonary valve stenosis (PS), repaired tetralogy of Fallot (ToF), chronic pulmonary regurgitation, intra- or extracardiac shunts, Ebstein's disease and transposition of great arteries with systemic RV (Figs. 2, 3 and 4) [10-12].

\section{Tetralogy of Fallot}

ToF is a complex malformation characterised by four heart defects, including ventricular septal defect, an overriding aorta, PS and RV hypertrophy.

The disease is commonly repaired by closure of the ventricular septal defect and correction of the RV outflow tract stenosis with patches or valvotomy, resulting in a distortion of anatomy.

CMR is commonly required to rule out post-repair complications which variably may consist of residual atrial and/or ventricular septal defect, akinesis or dyskinesis of the right ventricular outflow tract, outflow patch aneurysm and fibrosis of RV free wall or pulmonary artery stenosis [12-15].

Pulmonary valve regurgitation is a nearly universal complication, accurately and reliably quantified by CMR using velocity-encoded phase-contrast technique, the degree of which has been demonstrated to be related to RV impairment, dilation or hypertrophy [13].

Velocity-encoded phase-contrast technique may also be useful to evaluate the trans-stenosis pressure gradient, by measuring peak velocity, when stenosis of RV outflow tract
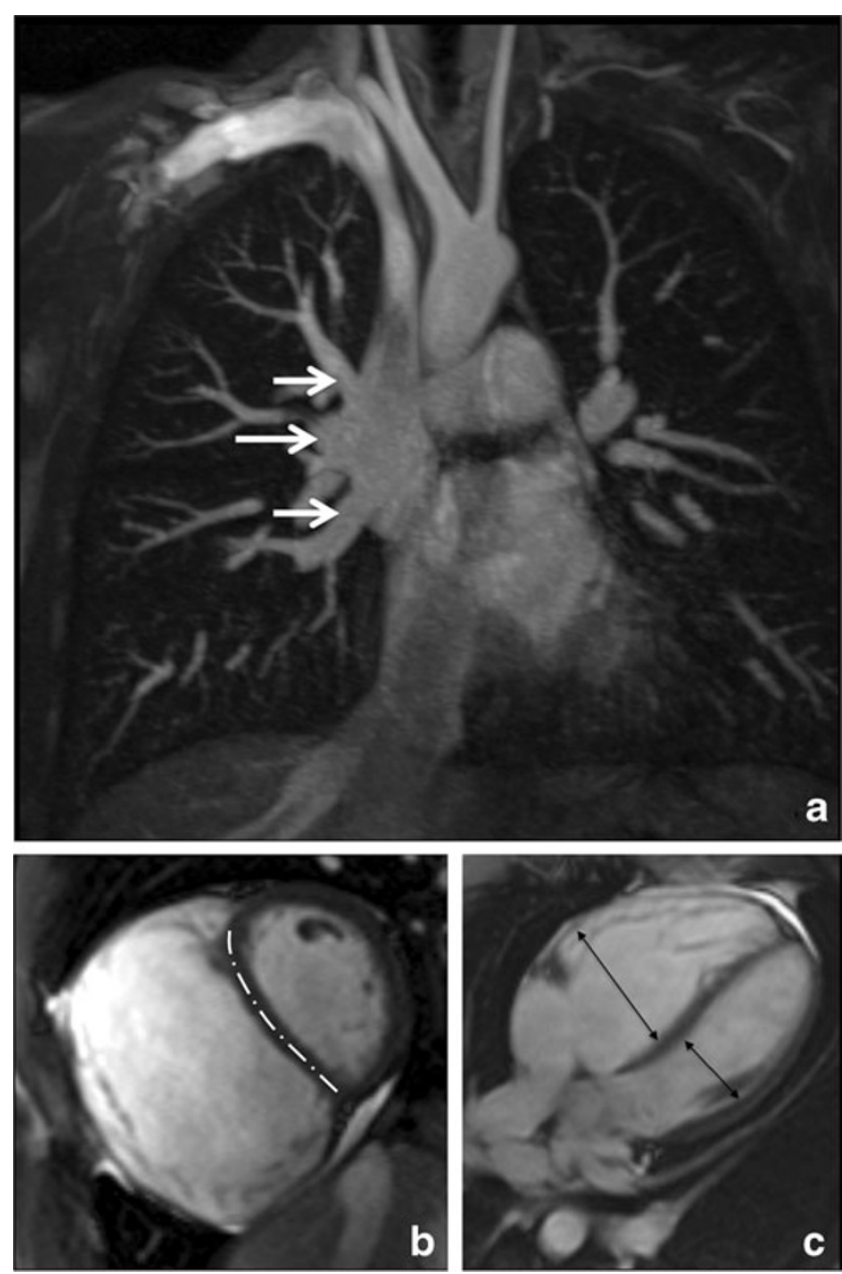

Fig. 3 A 29-year-old woman with partial anomalous pulmonary venous return: CMR evaluation was required to rule out secondary causes of pulmonary hypertension. Patient was asymptomatic. a MR angiography subtracted image shows anomalous drainage of both superior and inferior right pulmonary veins (white arrows) into the superior vena cava with subsequent signs of RV volume overload on cine-SSFP (b, c RV enlargement+ septal flattening) without hypertrophy reflecting low pulmonary vascular resistance

or pulmonary artery on cine-MR or MR angiogram images is detected [13].
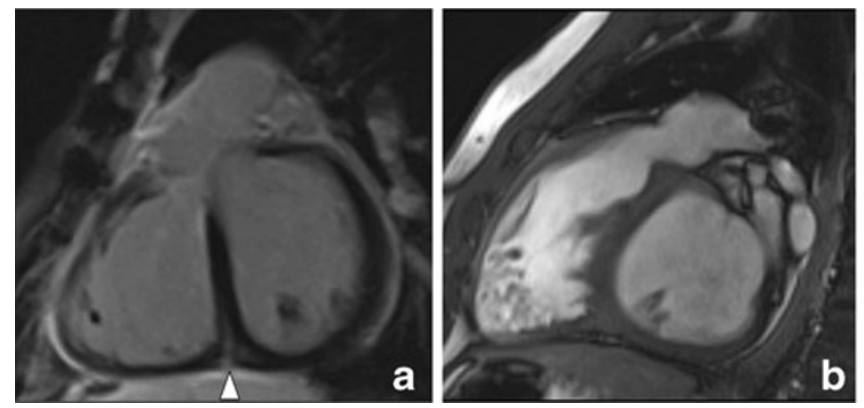

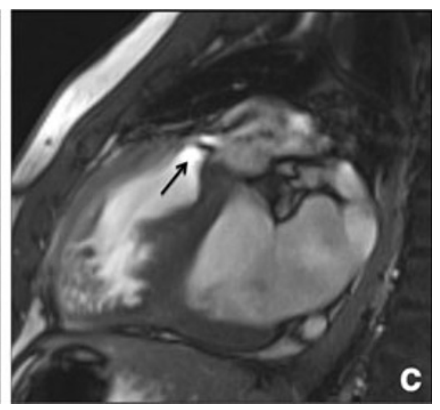

systole (c) reveal subvalvular pulmonary residual stenosis (black arrow) and significant right ventricular myocardial hypertrophy due to increased subvalvular flow resistance
Fig. 2 A 35-year-old man with surgically corrected tetralogy of Fallot. tern characterised by a focal area at the inferior ventricular junction (arrowhead); cine SSFP short-axis images during diastole (b) and

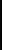




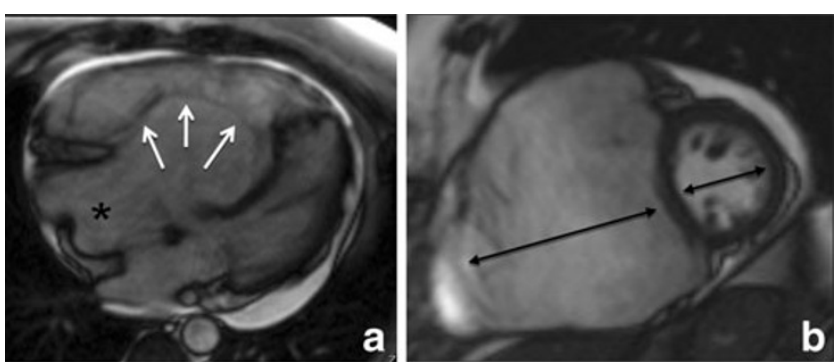

Fig. 4 A 12-year-old boy with Ebstein anomaly: cine SSFP fourchamber view (a) shows the anomalous position of the tricuspid valve (white arrows) with leftward displacement of the septum due to RV overload; moderate pericardial effusion is also present; asterisk right atrium. Cine SSFP short-axis view (b) demonstrates huge right chamber enlargement compared with left ventricle (black arrows)

Furthermore, in patients with corrected ToF, dys-synchrony of RV contraction or abnormal excursion of interventricular septum, easily recognised on cine MR images, contribute to RV dysfunction and are often associated with fibrosis at the interventricular junction points, suggesting a mechanism of adverse interventricular interaction (Fig. 2) [14, 15].

\section{Transposition of great arteries}

In patients with congenitally corrected transposition or transposition that has been corrected with atrial switch, the morphological RV pumps blood to the aorta.

For these patients, the accurate measurement of ventricular volumes obtained by CMR is useful in assessing adaptive modification to systemic resistance leading to $\mathrm{RV}$ dilation and hypertrophy $[10,11]$.

In patients treated with arterial switch, the RV may appear normal due to the corrected atrio-ventricular-arterial discordance; however, CMR is performed not only to evaluate biventricular function and morphology but also to rule out potential complications in the vessels, patches or valves $[10,11]$.

\section{Ebstein's anomaly}

Ebstein's anomaly is a rare malformation of the tricuspid valve, characterised by the dilatation and apical displacement of the insertion of the septal leaflet and a redundant, elongated anterior leaflet (Fig. 4).

By definition, a part of the RV is anatomically ventricular but functionally atrial ("atrialised") and entirely contains the tricuspid valve. The remaining functional RV is small.

This anomaly can be associated to a variable degree of tricuspid regurgitation and RV dysfunction, progressively leading to a considerable right atrial and ventricular enlargement [16-18].

CMR, using stacks of cine sequences acquired on shortaxis, four-chamber and a RV vertical long-axis (RV inflowoutflow plane), allows measurement of the size and systolic function of the right chambers even in cases of severe disease with marked distortion of the RV anatomy [16].

The right atrium and atrialised RV are remarkably enlarged when the sum of the right atrium and atrialised $\mathrm{RV}$ area is larger than the combined area of the functional $\mathrm{RV}$, left atrium and left ventricle, measured on four-chamber view at the end diastole [17].

Moreover, the severity of tricuspid regurgitation can be estimated using a through-plane velocity-encoded sequence, oriented perpendicular to the regurgitant jet immediately on the atrial side.

A jet cross-sectional area equal or greater than $6 \times 6 \mathrm{~mm}$, setting $250 \mathrm{~cm} / \mathrm{s}$ as encoding velocity, can be regarded as severe [18].

An atrial septal defect occurs in about $50 \%$ of adult Ebstein patients and should be ruled out on atrial shortaxis cine images [18].

\section{Ischaemic cardiac disease}

Involvement of the RV occurs in approximately $50 \%$ of patients with inferior myocardial infarction (MI), whereas signs of RV ischaemia have been reported with CMR in around $33 \%$ of anterior LV MI [4, 19-21]. Increasing interest in non-invasive recognition of RV MI is justified by its prognostic and therapeutic implications (adverse RV remodelling and worsening of functional recovery at followup). These patients have higher rates of in-hospital morbidity and mortality than isolated LV infarction, thus requiring more aggressive treatment approaches $[3,19,20]$. A significant discrepancy, however, between the frequency of RV MI at autopsy and detected cases amongst living patients demonstrates an underestimation of the phenomenon by routine clinical and instrumental criteria (electrocardiography, echocardiography, haemodynamic monitoring)[21].

CMR represents an ideal tool for its detection in both acute and chronic MIs although the thinned infarcted wall of the RV (mostly in chronic lesions) is not always easily detected with either T2WI and LGE-T1WI sequences [4].

Typically, necrosis extends from the LV inferior wall and septum to the posterior RV wall and is caused by the occlusion of the right coronary artery (in the case of right dominance) or circumflex artery (left dominance) (Fig. 5) [19]. However, ischaemic injury affects RV anterolateral free wall in a considerable number of patient with anterior LV MI, by occlusion of anterior descending artery or its branches (Table 1).

Right myocardium is less susceptible to ischaemic injury compared with left, due to:

- Lower demand for oxygen due to lower contractile function

- Greater oxygen reserve during hemodynamic stress 

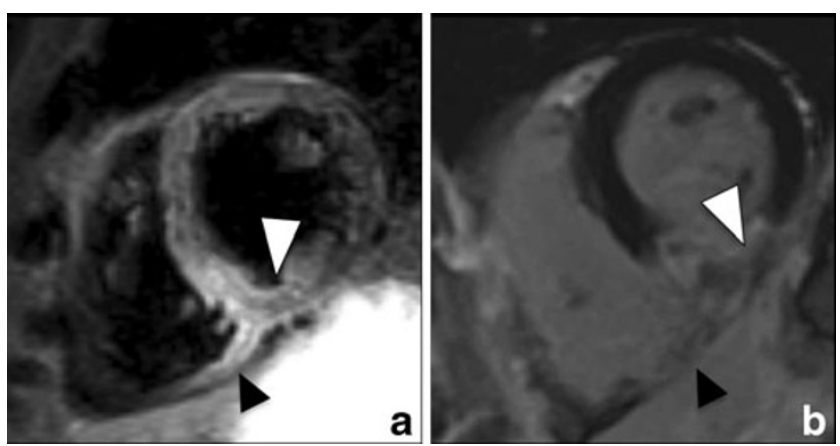

Fig. 5 A 57-year-old man with inferior myocardial infarction assessed in the acute phase at the 5th day after reperfusion of right coronary artery. T2-weighted short-axis image (a) demonstrates tissue oedema located within the mid inferior LV wall (white arrowhead) with extension to the medial-inferior wall of the RV (black arrowhead). Corresponding short-axis LGE image (b) shows transmural LGE involving same segments (arrows)

- The peculiarity of the right coronary circulation (perfusion during both systole and diastole, and more extensive collateral flow) $[3,19]$

Right ventricular dilatation, abnormal right ventricular wall motion, paradoxical motion of septum and tricuspid regurgitation are typical CMR features of RV MI, although $\mathrm{RV}$ transient dysfunction is frequently caused by a postinfarct stunning of RV myocardium in a LV MI [3].

A combined T2-weighted imaging/LGE imaging CMR approach enables the characterisation of reversibly and irreversibly damaged myocardium, and monitoring the progression from acute to chronic RV infarction (Fig. 5) [4].

\section{Non-ischaemic cardiomyopathy}

Arrhythmogenic right ventricular cardiomyopathy/dysplasia

Arrhythmogenic right ventricular cardiomyopathy (ARVC) is an inherited cardiomyopathy, predisposing patients to ventricular arrhythmias and sudden cardiac death. It is characterised by structural and functional abnormalities of the right ventricular myocardium, such as fibrofatty myocardium replacement, microaneurisms or focal atrophy [22].

Diagnosing ARVC is currently challenging due to the lack of an established and definite "gold standard" test, even including endomyocardial biopsy.

Although the revised criteria proposed by the Task Force of the European Society of Cardiology and the Scientific Council on Cardiomyopathies of the International Society and Federation of Cardiology in 2010 [23] confirmed the crucial role of CMR in diagnosing ARVC, a vigorous debate on the appropriate indications to CMR in patients with suspected ARVC is still ongoing.

CMR features may co-exist in the presence of global or segmental dilation of RV, reduced RV ejection fraction with normal or only mild LV impairment, localised RV aneurysms,

Table 1 MR features of right ventricular myocardial infarction

\begin{tabular}{|c|c|c|c|c|c|}
\hline $\begin{array}{l}\text { MYOCARDIAL } \\
\text { INFARCTION } \\
\text { PATTERN }\end{array}$ & $\begin{array}{l}\text { LV INFARCTION } \\
\text { LOCATION }\end{array}$ & $\begin{array}{l}\text { RV INFARCTION } \\
\text { INVOLVEMENT }\end{array}$ & INCIDENCE & $\begin{array}{l}\text { INFARCT-RELATED } \\
\text { ARTERY }^{\star}\end{array}$ & LGE PATTERN \\
\hline ANTERIOR MI & $\begin{array}{l}\text { Anterior wall and } \\
\text { anterior portion } \\
\text { of septum }\end{array}$ & $\begin{array}{c}\text { Anterior / antero-lateral } \\
\text { portion of RV free wall } \dagger \\
\text { and/or } \\
\text { RV Apex } \dagger\end{array}$ & $\begin{array}{l}\text { Around } 33 \% \text { of } \\
\text { anterior LV MI a }\end{array}$ & $\begin{array}{l}\text { Proximal left anterior } \\
\text { descending artery }\end{array}$ & \\
\hline INFERIOR MI & $\begin{array}{l}\text { Inferior wall and } \\
\text { inferior portion } \\
\text { of septum }\end{array}$ & $\begin{array}{c}\text { Inferior / infero-lateral } \\
\text { portion of RV free wall } \dagger\end{array}$ & $\begin{array}{l}\text { Around } 50 \% \text { of } \\
\text { inferior LV MI o }\end{array}$ & $\begin{array}{l}\text { Right coronary artery } \\
\text { (dominant) }\end{array}$ & \\
\hline $\begin{array}{l}\text { ISOLATED RIGHT } \\
\text { VENTRICULAR MI }\end{array}$ & $\begin{array}{l}\text { None } \\
\text { (septum is spared) }\end{array}$ & $\begin{array}{l}\text { Lateral and/or inferior } \\
\text { portion of RV free wall }\end{array}$ & $\begin{array}{c}\text { Unknown } \\
\text { (described only as } \\
\text { isolated cases) }\end{array}$ & $\begin{array}{l}\text { Right coronary artery } \\
\text { (non dominant) } \\
\text { or selective RCA } \\
\text { branches occlusion }\end{array}$ & \\
\hline
\end{tabular}


regional RV akinesia or dyskinesia and dys-synchronous RV contraction (Table 2) [23].

Recognition of fibrofatty myocardium replacement on CMR images is not a specific feature for ARVC, as it is frequently observed in elderly patients as part of the ageing process or as result of healed MI [24].

Moreover, differentiating myocardial from epicardial fat is often problematic due to the limited intrinsic spatial and contrast resolution of CMR. Notably, in ARVC, fat infiltration has been described to extend from the epicardium towards the endocardium; hence the RV free wall is remarkably thin. In healed MI, conversely, fat is band-like and mainly located in the subendocardial or mid-wall myocardium. On the other hand, RV free wall maintains normal or increase thickness in healthy patients with physiological fat [24].

In order to distinguish between LGE and fat, and to differentiate pathological fatty infiltration from the epicardial fat, TSE sequences ("black blood") should be acquired with and without fat saturation covering the entire volume of RV (Table 2).

The use of gadolinium in cases of suspected ARVC may be justified by the possible evidence of late enhancement related to fibrous degeneration in RV myocardium, with special focus to the so-called "triangle of dysplasia" (including diaphragmatic, apical and infundibular regions) [25].

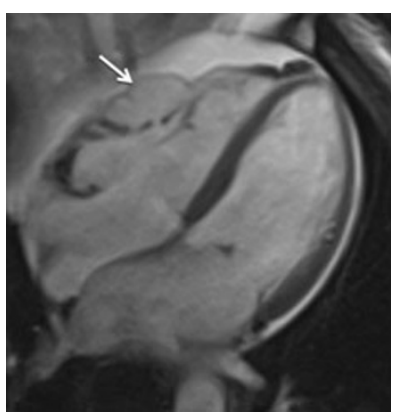

Fig. 6 Arrhythmogenic right ventricular dysplasia in a 38-year-old man presenting with syncope and familiar history of sudden cardiac death. Cine SSFP four-chamber image demonstrates multiple aneurysms (arrow) of RV free wall, that appears corrugated ("accordion sign")

Additionally, a typical morphological feature is the corrugation of right ventricular free wall, known as the "accordion sign" (Fig. 6) [25].

Hypertrophic cardiomyopathy

Hypertrophic cardiomyopathy (HCM) is the most common hereditary heart disease that usually affects the LV and can cause sudden cardiac death and heart failure at any age.

Notwithstanding the fact that the RV has long been considered spared by HCM, the introduction of CMR has

Table 2 MR features of arrhythmogenic right ventricular cardiomyopathy/dysplasia

\section{Revised Task Force MR Criteria* \\ Major Criteria \\ Regional RV akinesia or dyskinesia or dyssynchronous RV contraction \\ and 1 of the following: \\ - Ratio of RV end-diastolic volume to BSA $2110 \mathrm{~mL} / \mathrm{m} 2$ (male) or $2100 \mathrm{~mL} / \mathrm{m} 2$ (female) \\ - RV ejection fraction $540 \%$ \\ Minor criteria \\ Regional RV akinesia or dyskinesia or dyssynchronous RV contraction \\ and 1 of the following: \\ - Ratio of RV end-diastolic volume to BSA : 100 to $110 \mathrm{~mL} / \mathrm{m} 2$ (male) or 90 to $100 \mathrm{~mL} / \mathrm{m} 2$ (female) \\ - RV ejection fraction $40 \%$ to $45 \%$ \\ Recommended sequences \\ Additional MR features (non criteria) \\ Saccular RV aneurysms at the apex and inferior wall (akinetic or dyskinetic areas with diastolic bulging) \\ RV fibrofatty myocardial replacement (free wall, inferior wall, outflow) \\ Myocardial fat in the RV trabeculations, moderator band, interventricular septum and epicardial LV free wall $\dagger$

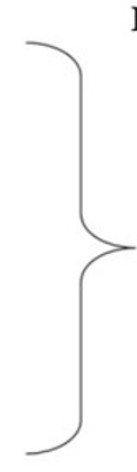 \\ b-SSFP cine-MR \\ (short-axis, horizontal and \\ vertical long-axis) \\ b-SSFP cine-MR \\ TSE T1w; fs-TSE T1w; LGE \\ T1w \\ TSE T1w; fs-TSE T1w}

$R V$ right ventricle, $B S A$ body surface area, $L V$ left ventricle, $b$-SSFP cine-MR balanced steady state free precession cine-MR sequence, $T S E T I w$ turbo spin echo T1-weighted sequence, $f S-T S E T 1 w$ fat suppressed turbo spin echo T1-weighted sequence, $L G E$ T1w late gadolinium-enhanced inversion recovery $\mathrm{T} 1$-weighted sequence

* Readapted by Marcus et al. [23] — Definite diagnosis is made when two major criteria, or one major and two minor criteria, or four minor criteria are met from different categories (Global or regional dysfunction and structural alterations, Tissue characterization of wall, Repolarisation abnormalities, Depolarization/conduction abnormalities, Arrhythmias, Family history)

$\dagger$ See [24] 

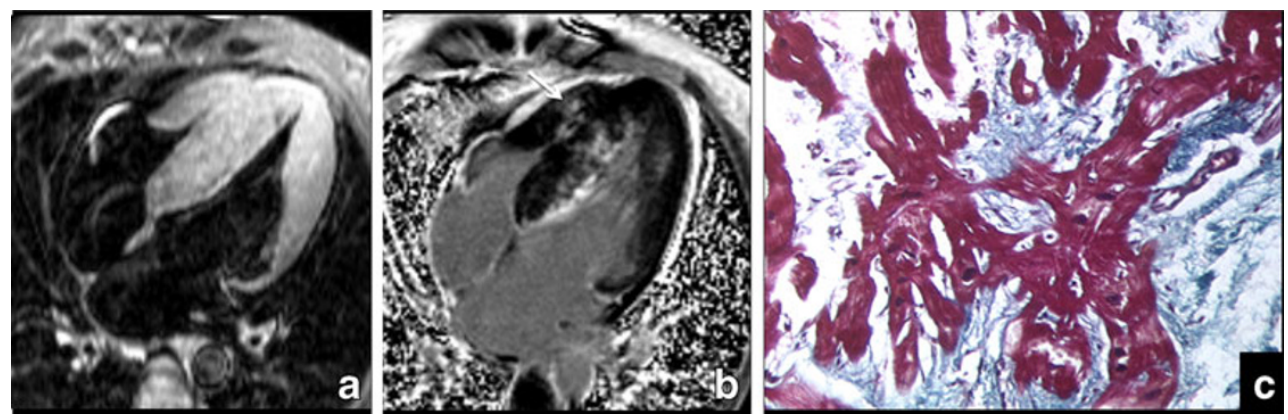

Fig. 7 Biventricular hypertrophic cardiomyopathy in a 37-year-old woman with history of syncope and fatigue. Severe concentric biventricular hypertrophy (septal thickness $27 \mathrm{~mm}$; LV mass $389 \mathrm{~g}$ ) with volumetric reduction of ventricular cavities and inhomogeneous biventricular spotty areas of signal hyperintensity are depicted on T2WI four-chamber (a) view reflecting diffuse myocardial oedema;

revealed an incidence of RV involvement at around $33 \%$ (if considered RV wall thickness $>8 \mathrm{~mm}$ ) among HCM patients population [26]. It may result in a RV outflow obstruction and/or an impaired RV diastolic filling with potentially severe dyspnea, supraventricular arrythmias and pulmonary thromboembolism

Patterns of RV hypertrophy range from a diffused form to hypertrophy of RV myocardium contiguous to interventricular septum to a confined focal thickening of apical RV myocardium (Fig. 7) [27]. A typical and highly specific feature, however, is the hypertrophy of the crista supraventricularis muscle.

Occasionally, myocardial disarray of the right ventricular myocardium can be revealed on LGE imaging and can indicate a potential arrhythmogenic substrate and its extent reflects the severity of the disease [28].

In the quantitative assessment of RV function, ejection fraction is usually similar to normal subjects, whereas enddiastolic volume is slightly lower due to diastolic filling dysfunction (likewise to functional changes observed in hypertrophied LV); this observation may suggest a unique primary hypertrophic process developing similarly in both ventricles [26] late enhancement image (b) shows extensive tissue damage with late enhancement involving both ventricles including the RV free apical wall (arrow). c Endomyocardial biopsy image confirms a severe hypertrophy and disarray of myocardiocites interrupted in short runs by replacement fibrosis (Masson Trichrome, 400×)

Non-compaction cardiomyopathy

Non-compaction cardiomyopathy (NCCMP) is characterised by a spongy appearance of the ventricular myocardium due to multiple, prominent trabeculations and deep intertrabecular recesses in communication with the ventricular cavity.

Typically, non-compaction involves the apical portion of the LV and predisposes to thromboemboli, arrhythmias, sudden death, ventricular dysfunction and heart failure.

CMR identifies left ventricular NCCMP with a sensitivity of $86 \%$ and a specificity of $99 \%$ using a cut-off ratio of non-compacted/compacted myocardium $>2.3$, measured on cine images at end-diastole [29].

However, RV non-compaction has been rarely described [30], since shared criteria to distinguish between normal RV trabeculations and non-compacted pattern has not yet been codified, thus making findings easily undiagnosed by inexperienced readers.

Left ventricular LGE was depicted in patients with NCCMP according to the presence of fibrosis, and recently
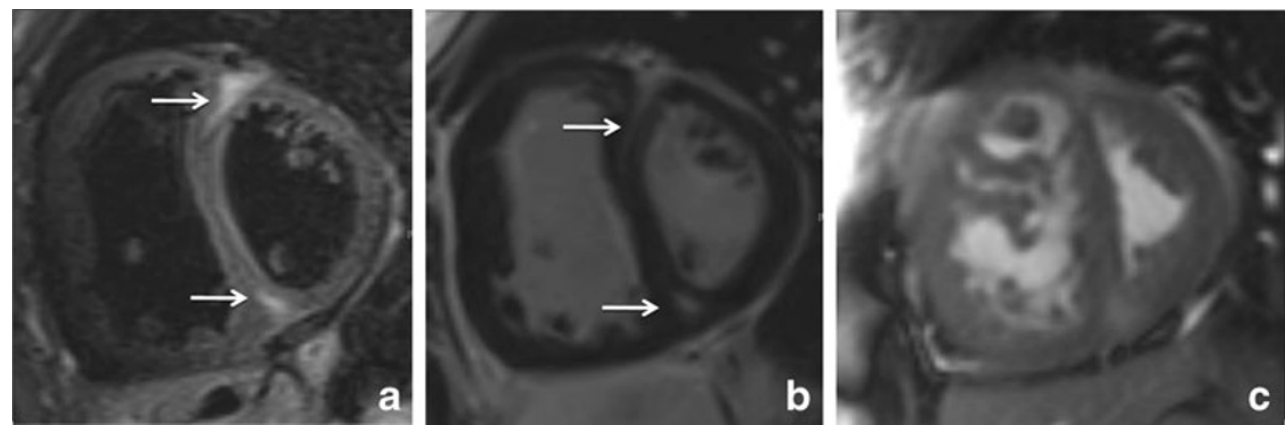

Fig. 8 Severe idiopathic pulmonary hypertension in a 28 year-old man; pulmonary arterial pressure at right catheterisation was $70 \mathrm{mmHg}$. Focal areas of hyperintense signal are observed at the level of both ventricular junctions on STIR T2WI (a, arrows) matching mesocardial spots of late enhancement in the same location (b, arrows). Severe concentric RV hypertrophy is observed on short-axis cine-SSFP endsystole image with flattening and inversion of the interventricular septum during contraction (c) 
demonstrated to be correlated to clinical severity and ventricular dysfunction [31].

CMR provides unique diagnostic clues to rule out this uncommon clinical entity due to its superior morphological depiction, which allows to objectively and reproducibly identify the extent of pathological hypertrabeculations, to depict eventually associated regional kinetic abnormalities and to exclude endocavitary thrombi.

\section{Cor pulmonale}

Cor pulmonale is defined as dilation and hypertrophy of the $\mathrm{RV}$ as a response to elevated pulmonary arterial pressure (pulmonary hypertension $[\mathrm{PH}]$ ).

Although a large spectrum of recognised conditions can cause cor pulmonale, ranging from heritable disorders to chronic pulmonary thromboembolism or lung disease, the aetiology remains unknown in a large number of cases (idiopathic pulmonary arterial hypertension) (Fig. 8).

CMR with angiographic visualisation of thoracic vessels allows, in a one-step shot, to:

- Evaluate functional and hemodynamic impairment of RV

- Detect vascular abnormalities, hypertrophy and fibrotic myocardial replacement of RV (Fig. 9) [32]

Typical CMR features of PH are RV dilation with unusual hypertrophy, right atrium dilation, flattened or reversed septum curvature (bulging) with dyskinetic movement [15]. LGE imaging shows a characteristic retention of gadolinium at the septal insertion (junctional pattern), predicting RV remodelling in response to increased afterload [32].

Initial and predictive signs of $\mathrm{PH}$ at $\mathrm{CMR}$ are considered to be a slowed and turbulent flow-pattern of the pulmonary artery. It has recently been utilised in the measurement of the degree of whirling flow on phase-contrast imaging and

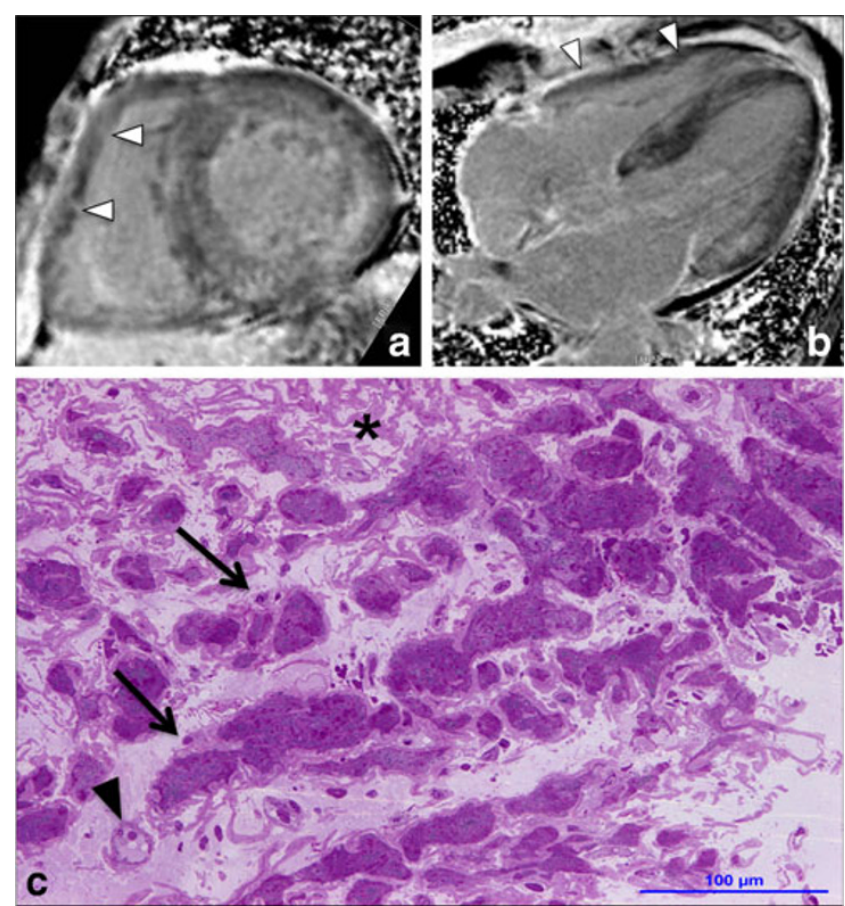

Fig. 10 Primary biventricular amyloidosis in 41-year-old woman addressed to CMR for characterisation of an unexplained LV concentric hypertrophy and to rule out hypertrophic cardiomiopathy. A concentric LV wall thickening with RV involvement (white arrowheads) and the characteristic diffuse heterogeneous "non-suppressed" appearance of the myocardium is depicted on late-enhanced phase sensitive inversion recovery T1-weighted short axis (a) and four-chamber view images (b). c Endomyocardial biopsy demonstrated extensive amyloid substance occupying the interstitium (asterisk), surrounding myocardiocites (arrows) and small vessels (black arrowhead)

the distensibility of the pulmonary trunk on the cine CMR images by comparing systolic and diastolic transverse diameters $[33,34]$.

Other non-ischaemic cardiomyopathies

The RV can be involved in various, other cardiac diseases (e.g. dilated cardiomyopathy, infective or
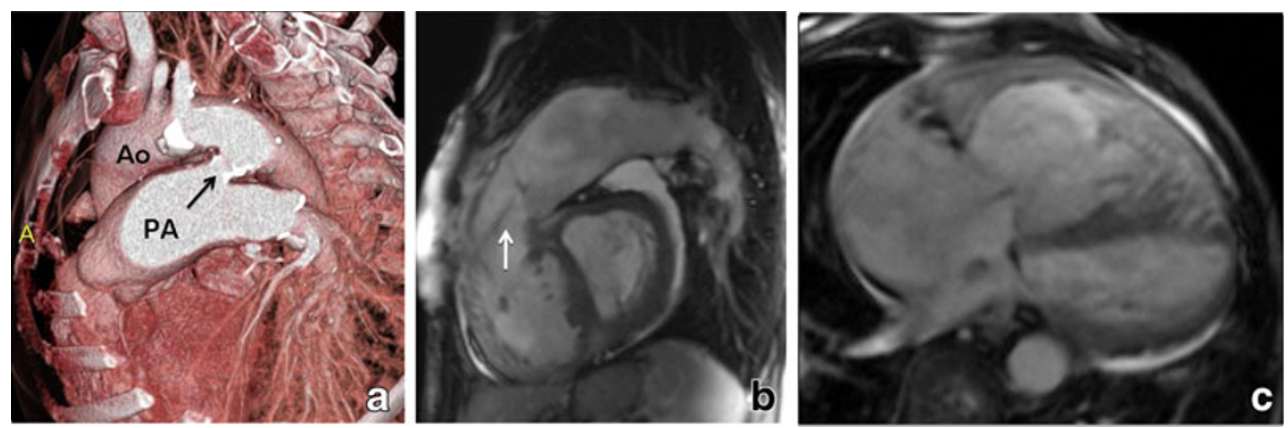

Fig. 9 Secondary pulmonary hypertension related to patent ductus arteriosus in a 32-year-old man presenting with fatigue and stiffness. a Volume rendering reconstruction of CT Angiography shows a communication between aortic arch $(A o)$ and pulmonary trunk $(P A)$ for the presence of patent ductus arteriosus (black arrow). b, c In the same patient, cine-SSFP images demonstrate dilated pulmonary artery with valvular regurgitation (white arrow, b) and severe eccentric RV enlargement at four-chamber view (c) 
autoimmune myocarditis, Anderson Fabry disease [35]) or associated with systemic disorders (e.g. amyloidosis, sarcoidosis, Wegener's granulomatosis [36] or storage disease) (Figs. 10 and 11).

In the most of these conditions, however, the CMR features resemble the LV pattern, as observed in several case reports.

\section{Right ventricular masses}

The masses most frequently encountered in the RV are non-tumoral, such as thrombi, valvular vegetations, focal muscular hypertrophy and pericardial cysts but despite their non-neoplastic nature they may lead to severe complications (embolisation, ventricular function impairment) [37].
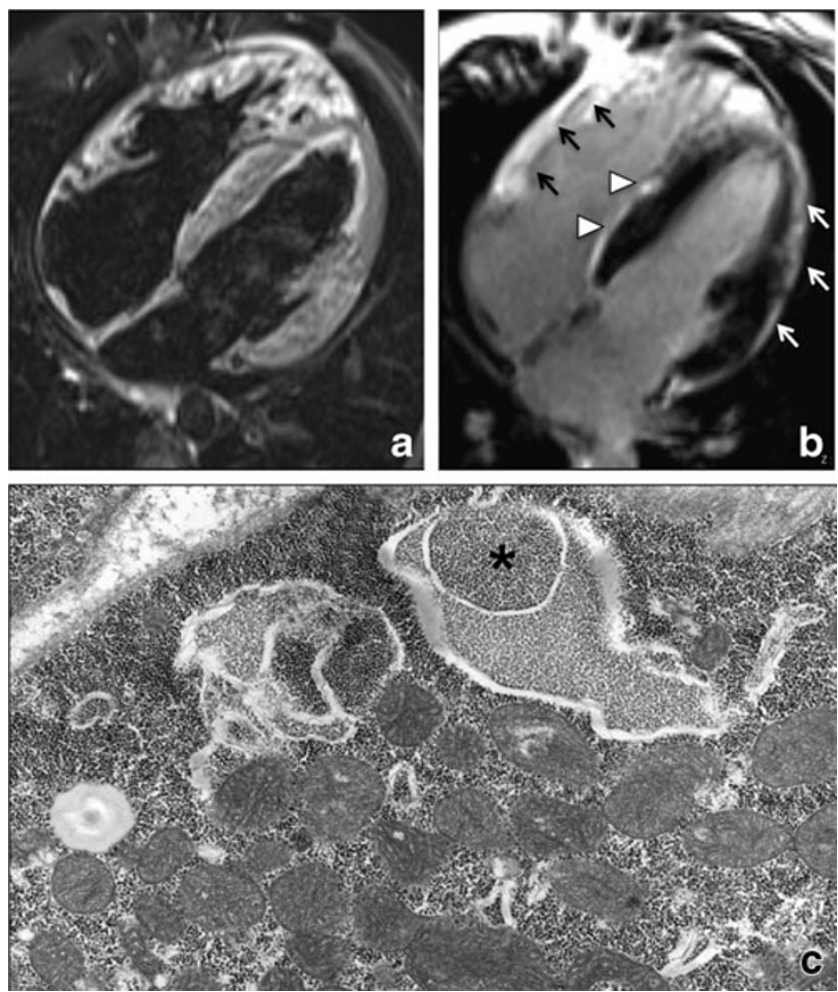

Fig. 11 Glycogen storage disease in a 29-year-old man with family history of juvenile sudden cardiac death and unexplained increased left ventricular wall thickness at echocardiography. a. Four-chamber T2WI shows an hyper-trabeculated appearance of the RV with subendocardial hyperintense signal related to "slowflow" phenomenon caused by diffused hypokinesis. b. Four-chamber LGE-T1WI reveals a diffuse and homogeneous enhancement of the RV myocardium (black arrows) involving the free wall, apex, and right side of the interventricular septum (arrowheads). The late-enhancement pattern of the left ventricle is predominantly subepicardial (white arrows) involving the lateral wall. c. Electron microscopy image of endomyocardial biopsy shows massive accumulation of citosolic glycogen, sometimes engulfing autophagosoms (asterisk)

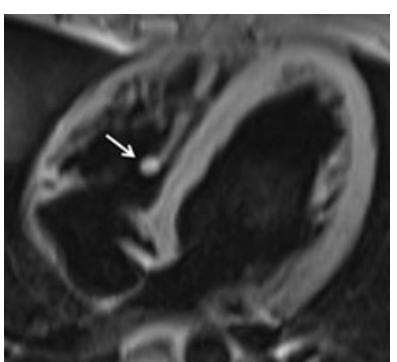

Fig. 12 RV fibroelastoma in an asymptomatic 32-year-old man with an intracavitary nodule incidentally depicted at trans-thoracic echocardiography. TSE T1-weighted image shows the presence of a nodular rounded RV mass (arrow) attached to a large trabecula

RV implication in primary cardiac tumours is extremely rare and the true incidence varies on the different series, being agreed that benign lesions (e.g. myxoma, lipoma, papillary fibroelastoma, rhabdomyoma, fibroma) occur 3-4 times more frequently than primary malignancies (e.g. angiosarcoma, rhabdomyosarcoma, liposarcoma, lymphoma, etc.) (Figs. 12 and 13) [38]: CMR features such as invasion of pericardium, regular edge and preservation of anatomical borders can be helpful in their distinction.

Finally, it has estimated that secondary cardiac tumours are a hundred times more common compared with primary lesions [39]; and the RV may be reached by direct extension such as (e.g. lung or breast cancer), lymphatic extension (e.g. lymphoma), or metastatic spread (e.g. malignant melanoma or renal cell carcinoma) [37].

\section{Conclusion}

CMR allows a comprehensive evaluation of the right ventricle both in terms of anatomical delineation, function quantification and tissue characterisation.

A proper knowledge of the most common CMR features of RV diseases is necessary in order to promptly and accurately identify the $\mathrm{RV}$ involvement in various cardiac diseases.

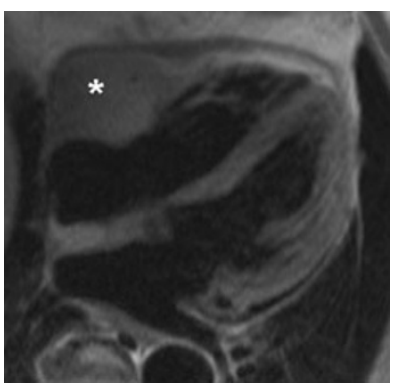

Fig. 13 RV metastasis of renal cancer in a 62-year-old man (histologically proven). A large ovoid secondary lesion is depicted at the lateral atrio-ventricular junction compressing the RV free wall 
Disclosure No grants have been received regarding this work.

The authors have no conflicts of interest to declare and have no direct or indirect financial interest in the subject matter of this manuscript.

Open Access This article is distributed under the terms of the Creative Commons Attribution License which permits any use, distribution, and reproduction in any medium, provided the original author(s) and the source are credited.

\section{References}

1. Rigolin VH, Robiolio PA, Wilson JS, Harrison JK, Bashore TM et al (1995) The forgotten chamber: the importance of the right ventricle. Catheter Cardiovasc Diagn 35(1):18-28

2. Kawut SM, Barr RG, Lima JA, Praestgaard A, Johnson WC et al (2012) Circulation 126:1681-1688

3. Goldstein JA (2002) Pathophysiology and management of right heart ischemia. J Am Coll Cardiol 40(5):841-853

4. Masci PG, Francone M, Desmet W et al (2010) Right ventricular ischemic injury in patients with acute ST-segment elevation myocardial infarction: characterization with cardiovascular magnetic resonance. Circulation 122(14):1405-1412

5. Marcu CB, Beek AM, Van Rossum AC et al (2006) Cardiovascular magnetic resonance imaging for the assessment of right heart Involvement in cardiac and pulmonary disease. Heart Lung Circ 15(6):362-370

6. Alfakih K, Reid S, Jones T, Sivananthan M et al (2004) Assessment of ventricular function and mass by cardiac magnetic resonance imaging. Eur Radiol 14(10):1813-1822

7. Sanz J, Conroy J, Narula J (2012) Imaging of the right ventricle. Cardiol Clin 30(2):189-203

8. Valsangiacomo Buechel ER, Mertens LL (2012) Imaging the right heart: the use of integrated multimodality imaging. Eur Heart J 33 (8):949-960

9. Krishnamurthy R (2009) The role of MRI and CT in congenital heart disease. Pediatr Radiol 39(Suppl 2):S196-S204

10. Warnes CA (2009) Adult congenital heart disease importance of the right ventricle. J Am Coll Cardiol 54(21):1903-1910

11. Davlouros PA, Niwa K, Webb G, Gatzoulis MA (2006) The right ventricle in congenital heart disease. Heart 92(Suppl 1): i27-i38

12. Van Straten A, Vliegen HW, Hazekamp MG, de Roos A (2005) Right ventricular function late after total repair of tetralogy of Fallot. Eur Radiol 15(4):702-707

13. Geva T (2011) Repaired tetralogy of Fallot: the roles of cardiovascular magnetic resonance in evaluating pathophysiology and for pulmonary valve replacement decision support. J Cardiovasc Magn Reson 13:9

14. Muzzarelli S, Ordovas KG, Cannavale G, Meadows AK, Higgins CB (2011) Impact of the excursion of the interventricular septum on left ventricular systolic function and fibrosis after surgical repair. Radiology 259(2):375-383

15. Méndez C, Soler R, Rodriguez E, López M, Álvarez L, Fernández N, Montserrat L (2011) Magnetic resonance imaging of abnormal ventricular septal motion in heart diseases: a pictorial review. Insights Imaging 2:483-492

16. Yalonetsky S, Tobler D, Greutmann M et al (2011) Cardiac magnetic resonance imaging and the assessment of ebstein anomaly in adults. Am J Cardiol 107(5):767-773

17. Celermajer DS, Bull C, Till JA, Cullen S, Vassillikos VP, Sullivan ID, Allan L, Nihoyannopoulos P, Somerville J, Deanfield JE (1994) Ebstein's anomaly: presentation and outcome from fetus to adult. J Am Coll Cardiol 23:170-176
18. Kilner PJ (2011) The role of cardiovascular magnetic resonance in adults with congenital heart disease. Prog Cardiovasc Dis 54(3):295-304

19. Kinch JW, Ryan TJ (1994) Right ventricular infarction. N Engl J Med 330(17):1211-1217

20. Mehta SR, Eikelboom JW, Natarajan MK, Diaz R, Yi C, Gibbons RJ, Yusuf S (2001) Impact of right ventricular involvement on mortality and morbidity in patients with inferior myocardial infarction. J Am Coll Cardiol 37(1):37-43

21. Isner JM, Roberts WC (1978) Right ventricular infarction complicating left ventricular infarction secondary to coronary heart disease. Frequency, location, associated findings and significance from analysis of 236 necropsy patients with acute or healed myocardial infarction. Am J Cardiol 42(6):885-894

22. McKenna WJ, Thiene G, Nava A et al (1994) Diagnosis of arrhythmogenic right ventricular dysplasia/cardiomyopathy. Task Force of the Working Group Myocardial and Pericardial Disease of the European Society of Cardiology and of the Scientific Council on Cardiomyopathies of the International Society and Federation of Cardiology. Br Heart J 71(3):215-218

23. Marcus FI, McKenna WJ, Sherrill D et al (2010) Diagnosis of arrhythmogenic right ventricular cardiomyopathy/dysplasia: proposed modification of the task force criteria. Circulation 121 (13):1533-1541

24. Kimura F, Matsuo Y, Nakajima T, Nishikawa T, Kawamura S, Sannohe S, Hagiwara N, Sakai F (2010) Myocardial fat at cardiac imaging: how can we differentiate pathologic from physiologic fatty infiltration? Radiographics 30:1587-1602

25. Murphy DT, Shine SC, Cradock A, Galvin JM, Keelan E, Murray JG (2010) Cardiac MRI in arrhythmogenic right ventricular cardiomyopathy. AJR Am J Roentgenol 194(4):W299-W306

26. Maron MS, Hauser TH, Dubrow E et al (2007) Right ventricular involvement in hypertrophic cardiomyopathy. Am J Cardiol 100 (8):1293-1298

27. Mozaffarian D, Caldwell JH (2001) Right ventricular involvement in hypertrophic cardiomyopathy: a case report and literature review. Clin Cardiol 24:2-8

28. Moon JC, Reed E, Sheppard MN et al (2004) The histologic basis of late gadolinium enhancement cardiovascular magnetic resonance in hypertrophic cardiomyopathy. J Am Coll Cardiol 43 (12):2260-2264

29. Petersen SE, Selvanayagam JB, Wiesmann F, Robson MD, Francis JM, Anderson RH, Watkins H, Neubauer S (2005) Left ventricular non-compaction insights from cardiovascular magnetic resonance imaging. J Am Coll Cardiol 46(1):101-105

30. Gomathi SB, Makadia N, Ajit SM (2008) An unusual case of isolated non-compacted right ventricular myocardium. Eur J Echocardiogr 9(3):424-425

31. Nucifora G, Aquaro GD, Pingitore A, Masci PG, Lombardi M (2011) Myocardial fibrosis in isolated left ventricular non-compaction and its relation to disease severity. Eur J Heart Fail 13(2):170-176

32. Shehata ML, Lossnitzer D, Skrok J et al (2011) Myocardial delayed enhancement in pulmonary hypertension: pulmonary hemodynamics, right ventricular function, and remodeling. AJR Am J Roentgenol 196(1):87-94

33. Sanz J, Kariisa M, Dellegrottaglie S, Prat-González S, Garcia MJ, Fuster V, Rajagopalan S (2009) Evaluation of pulmonary artery stiffness in pulmonary hypertension with cardiac magnetic resonance. JACC Cardiovasc Imaging 2(3):286-295

34. Sanz J, Kuschnir P, Rius T, Salguero R, Sulica R, Einstein AJ, Dellegrottaglie S, Fuster V, Rajagopalan S, Poon M (2007) Pulmonary arterial hypertension: noninvasive detection with phase-contrast MR imaging. Radiology 243(1):7079

35. Niemann M, Breunig F, Beer M et al (2010) The right ventricle in Fabry disease: natural history and impact of enzyme replacement therapy. Heart 96(23):1915-1919 
36. Miszalski-Jamka T, Szczeklik W, Sokolowska B et al (2011) Cardiac involvement in Wegener's granulomatosis resistant to induction therapy. Eur Radiol 21(11):22972304

37. Bogaert J, Dymarkowski S, Taylor AM (2005) Clinical cardiac MRI. Springer. Springer, Berlin Heidelberg
38. Yu K, Liu Y, Wang H, Hu S, Long C (2007) Epidemiological and pathological characteristics of cardiac tumors: a clinical study of 242 cases. Interact Cardiovasc Thorac Surg 6(5):636639

39. Roberts WC (1997) Primary and secondary neoplasms of the heart. Am J Cardiol 80(5):671-682 